\title{
Zur Kenntnis des Farbstoffes des antiken Purpurs aus Murex brandaris
}

von

\section{P. Friedlaender.}

(Vorgelegt in der Sitzung am 7. Jänner 1909.)

In einer früheren Mitteilung habe ich es auf Grund der Untersuchung einer kleinen Menge des Farbstoffes aus Murex brandaris als sehr wahrscheinlich hinstellen können, daß derselbe zu der Gruppe der sogenannten indigoiden Farbstoffe zu zählen sei. Es konnte ferner die Verschiedenheit sowohl vom Thioindigo wie vom Indigblau konstatiert werden.

Seither wurde ich durch eine Unterstützung der kaiserl. Akademie der Wissenschaften in Wien und durch das liebenswürdige Entgegenkommen einer Anzahl von in- und ausländischen Zoologischen Stationen, in erster Linie des Direktors der Zoologischen Station in Triest, Herrn Prof. Cori, ferner der Herren Dr. Hermes (Rovigno) und Prof. Dubois (Toulon), denen ich auch an dieser Stelle meinen besten Dank aussprechen möchte, in den Stand gesetzt, eine sehr viel größere Zahl von Schnecken, zirka 12.000, zu verarbeiten und verfügte schließlich über $1.4 g$ reinen Farbstoff, welche Menge für die Feststellung der Zusammensetzung und Konstitution sowie für die Synthese ausreichte.

Bei der Isolierung des Farbstoffes ergaben sich einige Modifikationen gegenüber dem früher beschriebenen Verfahren im Interesse eines expeditiveren Arbeitens als wünschenswert

Die herauspräparierten Drüsen von Murex brandaris wurden zunächst wie angegeben auf Filtrierpapier gestrichen 
und der Farbstoff durch kurzes Belichten in der Sonne entwickelt. Hierauf maceriert man das Papier durch $1 / 2$ stündiges Erwärmen auf dem Wasserbade mit mäßig verdünnter Schwefelsäure (1:2), saugt den Brei nach Zusatz von Wasser ab und wäscht mit heißem Wasser aus. Der Rückstand wird im Soxlethapparat so lange mit Alkohol ausgezogen, bis derselbe nicht mehr bräunlich gefärbt abläuft. Hierdurch werden der größte Teil der Verunreinigungen, zersetzte Eiweißsubstanzen, Fett, braun gefärbte schmierige Massen etc. entfernt, während der eigentliche Farbstoff intakt bleibt. Zur Isolierung desselben in der angegebenen Weise (Soxlethhülse, die in einem weithalsigen Kolben unterhalb des Kühlrohres aufgehängt wird) wurde ein höher siedendes Extraktionsmittel vorgezogen; ich blieb schließlich bei der Verwendung von Benzoesäureäthylester (Siedepunkt $210^{\circ}$ ), aber auch hierin ist Farbstoff so wenig löslich, daß sich beim Extrahieren von zirka $0.05 \mathrm{~g} \mathrm{mit} 1 / 2 \mathrm{l}$ Benzoesäureäther schon in der siedenden Flüssigkeit ein großer Teil in flinmernden Kryställchen ausscheidet. Beim Erkalten findet eine vollständige Abscheidung statt; das Lösungsmittel bleibt rötlichbraun gefärbt, aber die kupferglänzenden Kryställchen machen unter dem Mikroskop und bei Farbenreaktionen einen durchaus einheitlichen Eindruck; sie wurden mit Alkohol und Äther gewaschen und erwiesen sich bei der qualitativen Analyse als frei von Schwefel, Chlor und Jod, aber stickstoffhaltig und stark bromhaltig.

Die quantitative Analyse ergab folgende Zahlen:

I. $0.1011 \mathrm{~g}$ Substanz lieferten $0.1744 \mathrm{~g} \mathrm{CO}_{2}$ und $0.0208 g \mathrm{H}_{2} \mathrm{O}$.

II. $0.1023 g$ Substanz lieferten $0.1766 \mathrm{~g} \mathrm{CO}_{2}$ und $0.0218 \mathrm{~g} \mathrm{H}_{2} \mathrm{O}$.

III. $0.0824 \mathrm{~g}$ Substanz lieferten $0.0691 \mathrm{~g} \mathrm{Ag}$ Br.

IV. $0 \cdot 1316 \mathrm{~g}$ Substanz lieferten $0 \cdot 1112 \mathrm{~g} \mathrm{AgBr}$.

In 100 Teilen:

\begin{tabular}{|c|c|c|c|c|}
\hline & \multicolumn{4}{|c|}{ Gefunden } \\
\hline & I & II & III & IV \\
\hline & $47 \cdot 04$ & 47.08 & -- & - \\
\hline & $2 \cdot 28$ & $2 \cdot 36$ & - & - \\
\hline r. & - & - & $35 \cdot 70$ & $5 \cdot 9$ \\
\hline
\end{tabular}

Der Farbstoff wurde hierauf noch einmal aus Benzoesäureäther in der angegebenen Weise (mittels einer Soxleth- 
hülse) und dann aus siedendem Chinolin umkrystallisiert, mit Eisessig ausgekocht, mit Alkohol und Äther gewaschen und bei $120^{\circ}$ getrocknet. Farbenreaktionen und Aussehen erfuhren hierdurch keine Veränderung, doch verminderte sich die Quantität nicht unerheblich und die Ursache hierfür wurde leider erst später gefunden. Kocht man nämlich dünne Lösungen des Farbstoffes unter Luftzutritt, so verschwindet die Färbung ziemlich schnell. Dasselbe Verhalten zeigen auch Lösungen von Indigblau in siedendem Benzoe- oder Phtalsäureäther, Chinolin und anderen hoch siedenden Lösungsmitteln. Offenbar findet hier eine Oxydation des Farbstoffes bei der höheren Temperatur durch den Luftsauerstoff statt, wie sie von A. G. Perkin ${ }^{1}$ auch beim Sublimieren von Indigo unter Luftzutritt beobachtet wurde. Man kann diesen Verlusten durch Arbeiten in einer Kohlensäureatmosphäre begegnen.

Der aus Chinolin umkrystallisierte Farbstoff gab bei der Analyse folgende Zahlen:

$0.1186 \mathrm{~g}$ lieferten $0.1037 \mathrm{~g} \mathrm{AgBr}$.

$0.1104 \mathrm{~g}$ lieferten $7.0 \mathrm{~cm}^{3} \mathrm{~N}$ bei $21^{\circ}$ und $705 \mathrm{~mm}$.

$0.1201 \mathrm{~g}$ lieferten $0.1068 \mathrm{~g} \mathrm{AgBr}$.

$0.1503 \mathrm{~g}$ lieferten $0.2531 \mathrm{~g} \mathrm{CO}_{2}$ und $0.0288 \mathrm{~g} \mathrm{H}_{2} \mathrm{O}$.

In 100 Teilen:

\begin{tabular}{|c|c|c|c|}
\hline & \multicolumn{2}{|c|}{ Gefunden } & \multirow{2}{*}{$\begin{array}{r}\begin{array}{r}\text { Berechnet für } \\
\mathrm{C}_{16} \mathrm{H}_{8} \mathrm{Br}_{2} \mathrm{~N}_{2} \mathrm{O}_{2}\end{array} \\
\end{array}$} \\
\hline & I & II & \\
\hline$c \ldots \ldots$ & - & $45 \cdot 92$ & $45 \cdot 71$ \\
\hline $\mathrm{H} \ldots \ldots$ & - & $2 \cdot 13$ & $1 \cdot 90$ \\
\hline Br....... & $7 \cdot 40$ & $37 \cdot 78$ & $38 \cdot 09$ \\
\hline$N \ldots \ldots$ & $6 \cdot 63$ & - & $6 \cdot 67$ \\
\hline
\end{tabular}

Die Differenzen dieser Zahlen mit denen der ersten Analysen sind wohl weniger auf die größere Reinheit des Farbstoffes als auf den Umstand zurückzuführen, daß Benzoesäureäther ziemlich hartnäckig von dem Farbstoffe zurückgehalten wird, während sich Chinolin mit Eisessig leicht entfernen läßt.

Die gefundenen Zahlen lassen auf die Zusammensetzung $\mathrm{C}_{16} \mathrm{H}_{8} \mathrm{Br}_{2} \mathrm{~N}_{2} \mathrm{O}_{2}$ schließen und legen es nahe; den Farbstoff als

1 Proc. chem. soc., 22, 198. 
ein Dibromderivat des Indigotins oder des isomeren Indirubins aufzufassen, womit auch die beobachteten Eigenschaften im Einklange stehen würden. Nun sind nicht weniger als 50 isomere Dibromindigotine und Dibromindirubine theoretisch möglich und bei der geringen mir noch zur Verfügung stehenden Menge war es ganz ausgeschlossen, die Stellung der Bromatome etwa durch Abbąu $\mathrm{zu}$ einem Bromanilin oder einer Bromanthranilsäure $z u$ ermitteln, um so mehr als bei Indigo selbst die Überführung in Anilin oder Anthranilsäure keineswegs glatt gelingt.

Orientierende synthetische Versuche gestatteten jedoch mit großer Wahrscheinlichkeit zunächst die 28 möglichen Dibromindirubine auszuschließen. Die auf bekanntem Wege erhältlichen Farbstoffe wiesen nämlich in Nuance und Farbenreaktionen eine so große Differenz von dem Farbstoff aus Murex brandaris auf, daß die Zugehörigkeit zur Indirubingruppe äußerst unwahrscheinlich ist.

Aber auch von den 22 Dibromindigotinen scheidet auf Grund folgender Erwägung die Mehrzahl voraussichtlich aus: die in der frischen Drüse enthaltene Substanz, die am Licht in Farbstoff übergeht, ist in Lösungsmitteln wie Alkohol etc. leicht löslich. Wenn auch ihre Zusammensetzung noch unbekannt ist, darf man doch nach allen Erfahrungen auf diesem Gebiet annehmen, daß es sich um eine Verbindung vom halben Molekulargewichte $\mathrm{C}_{8} \mathrm{Br}$ handelt und daß sich bei der Farbstoffbildung $z$ wei gleiche Moleküle miteinander vereinigen. Mit dieser Annahme fallen aber 18 asymmetrisch substituierte Dibromindigotine fort (6 mononucleare, 12 binucleare) und es bleiben nur die vier symmetrischen binuclearen Farbstoffe, bei welchen man die Stellung der Bromatome am einfachsten durch Zahlen bezeichnet:

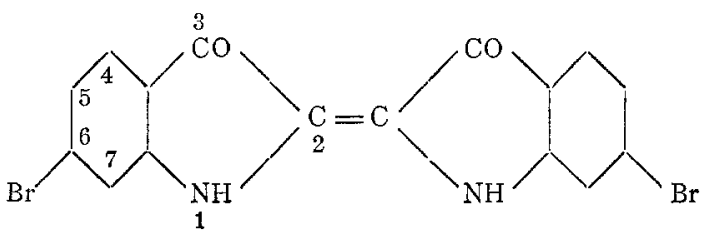

6.6-Dibromindigo 


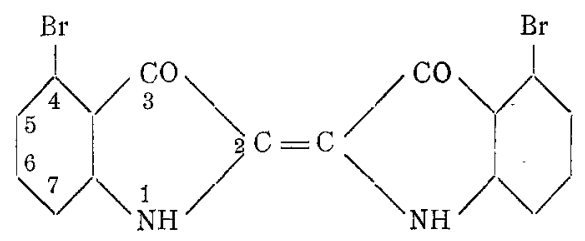

4.4-Dibromindigo

Von diesen ist 5.5-Dibromindigo bereits 1879 von A. v. B a e yer ${ }^{1}$ aus $m$-Bromisatinchlorid dargestellt worden; es unterscheidet sich von dem Murex-Farbstoff stark durch die sehr viel blaustichigere Nuance und durch noch schwerere Löslichkeit. Über 6.6-Dibromindigo findet sich eine kurze Notiz von Fr. Sachs und R. Kempf, ${ }^{2}$ die den Farbstoff aus $p$-Brom$o$-nitrobenzaldehyd erhielten. Die Angaben reichten zu einer eventuellen Identifizierung nicht aus und es erwies sich als notwendig, diese Farbstoffe sowie die noch nicht dargestellten isomeren 4.4- und 7.7-Dibromderivate nach bekannten Methoden zu synthetisieren und sie in Substanz zu vergleichen. Über Synthese und Eigenschaften dieser einzelnen Isomeren sowie verschiedener asymmetrisch substituierter Dibromindigotine, ferner über einige Dibromindirubine, die ich zusammen mit Herrn G. Deutsch darstellte, werde ich später berichten.

Der direkte Vergleich ergab dann die Identität des MurexFarbstoffes mit 6.6-Dibromindigo, der unter sämtlichen bisher untersuchten Isomeren die weitaus röteste Nuance besitzt. Eine bequeme Synthese gelang auf folgendem Wege: $p$-Toluidin wurde in bekannter Weise zu $o$-Nitro- $p$-Toluidin nitriert, in letzterem die $\mathrm{NH}_{2}$-Gruppe durch Brom ersetzt, die $\mathrm{NO}_{2}$-Gruppe $\mathrm{zu} \mathrm{NH}_{2}$ reduziert, die Acetylverbindung des entstehenden $p$-Brom-o-Toluidins mit Permanganat zu (Acetyl-) Bromanthranilsäure oxydiert, die sich mit Chloressigsäure in normaler Weise in das Glycinderivat überführen läßt. Zur Umwandlung in Bromindigo verwendet man Essigsäureanhydrid und wasserfreies Natriumacetat als Kondensationsmittel und oxydiert das entstandene Acetylbromindoxyl nach dem Verseifen mit Natronlauge durch Einblasen von Luft in alkalischer Lösung.

1 Ber. der Deutschen chem. Ges., 12, 1315.

2 Ibid. 36, 3303. 

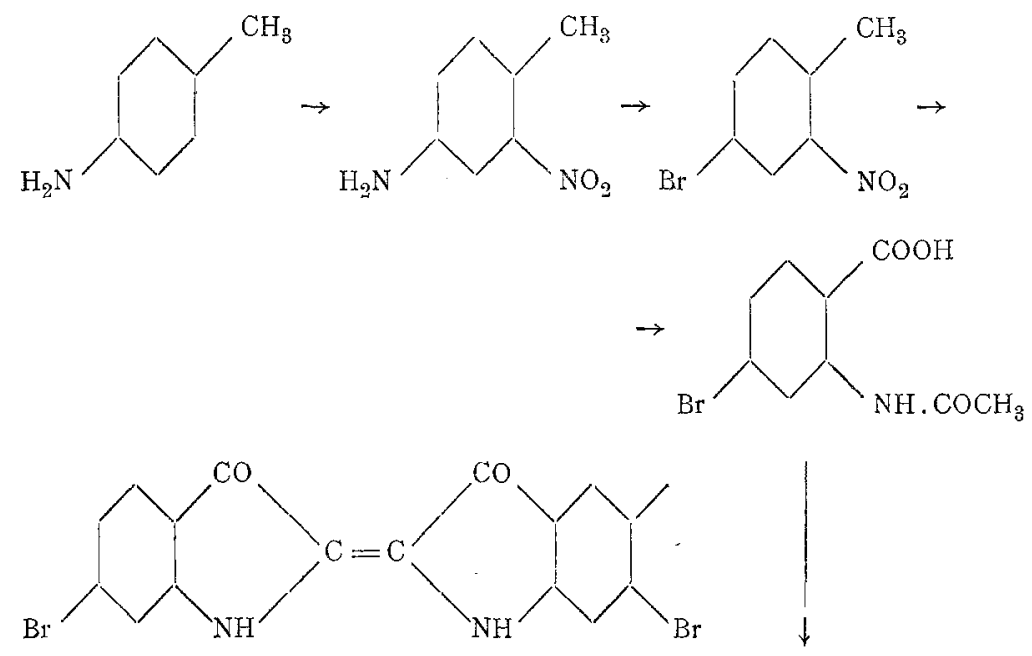

6.6-Dibromindigo

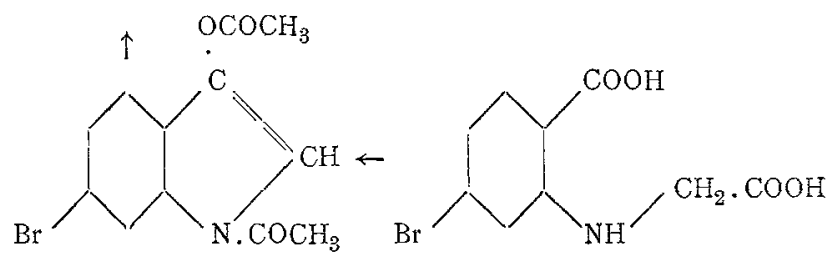

Der Farbstoff wurde hierauf in derselben Weise wie der aus Murex brandaris aus Benzoesäureäther und aus Chinolin umkrystallisiert. Zur näheren Charakterisierung seien folgende Angaben über Löslichkeitsverhältnisse und Farbenreaktionen angeführt, wobei eine Differenz zwischen den beiden Präparaten nicht konstatiert werden konnte.

Unlöslich in allen gebräuchlichen niedriger siedenden Lösungsmitteln, wie Alkohol, Äther, Benzol, Ligroin, Aceton etc.

Sehr schwer löslich in siedendem Pyridin mit rotvioletter, in siedendem Petroleum mit bläulichroter, in heißem Tetrachloräthan mit rotvioletter Nuance.

Etwas leichter löslich in siedendem Nitrobenzol, Anilin, Benzoesäureäther, noch leichter in siedendem Chinolin. Die Lösungen sind in dünneren Schichten blauviolett bis blau, in dickeren rotviolett durchscheinend. Beim Erkalten tritt vollständige Abscheidung ein. 
Konzentrierte Schwefelsäure löst in der Kälte nur sehr wenig mit braunvioletter Nuance, beim Erwärmen auf dem Wasserbade Farbenumschlag in ein etwas trübes Grün. Beim Eingießen in Wasser Abscheidung des unveränderten Farbstoffes in violettroten Flocken.

Schwefelsäurechlorhydrin löst in der Kälte ohne Veränderung mit charakteristischer rein kirschroter Farbe. Beim Erwärmen Farbenumschlag nach blau unter Bildung einer schwer löslichen blauen Sulfosäure; doch scheint die Sulfurierung, die unter denselben Erscheinungen auch mit rauchender Schwefelsäure erfolgt, nicht glatt zu verlaufen.

Bei Einwirkung von Natronlauge und Hydrosulfit entsteht eine schwach gelb gefärbte Küpe, aus der Baumwolle echt rotstichig violett gefärbt wird.

In spektroskopischer Hinsicht weisen gleich starke Lösungen der beiden Farbstoffe nach einer freundlichen Mitteilung von Hern Hofrat J. M. Eder keinen Unterschied auf.

Der weiteren Untersuchung bleibt es noch vorbehalten, festzustellen, ob andere im Altertum zum Färben benutzte Murex- und Purpura-Arten den gleichen Farbstoff liefern wie Murex brandaris. Bisher konnte ich nur konstatieren, daß bei der Belichtung der Drüsen von Murex truncultus neben einem rotvioletten gleichzeitig ein blauer Farbstoff entsteht, der mit Indigblau nicht identisch ist.

Es wird ferner von Interesse sein, die Natur der in der frischen Drüse enthaltenen Verbindung aufzuklären, die mit keiner der bis jetzt bekannten Indigo liefernden Substanzen Ähnlichkeit zu haben scheint. 